Pacific Journal of Mathematic 


\section{ON THE ENDOMORPHISM SEMIGROUP (AND CATEGORY) OF BOUNDED LATTICES}

\section{G. GRÄTZER AND J. SICHLER}

Recently, a large number of papers have been published on the representations of a semigroup with identity (i.e., a monoid) as the encomorphism semigroup of algebras and relational systems of various kinds. For instance $Z$. Hedrlín and J. Lambek showed that every monoid can be represented as the endomorphism semigroup of a semigroup.

Conspicuously missing from the list of algebras for which theorems of this type can be proved are lattices. The reason for this is that any constant map is a lattice endomorphism, and therefore endomorphism semigroups of lattices are very special. For partially ordered sets one can eliminate constant maps as endomorphisms by considering only those maps $\varphi$ that satisfy $x<y$ implies $x \varphi<y \varphi$ (Z. Hedrlín and R. H. McDowell); however, this would not be a very natural condition to impose on lattice endomorphisms.

The approach of this paper ${ }^{1}$ is to consider bounded lattices only, that is, lattices with smallest element 0 and largest element 1, and as endomorphisms to admit only those lattice endomorphisms that preserve 0 and 1 (i.e., keep 0 and 1 fixed; this amounts to considering 0 and 1 as nullary operations). Such endomorphisms are usually called $\{0,1\}$-endomorphisms but they are called simply endomorphisms in this paper.

The first result is that every monoid is isomorphic to the monoid of all endomorphisms of a bounded lattice.

One can also consider lattices with complementation $\left\langle L ; \wedge, \vee,{ }^{\prime}\right\rangle$, where ' is a complementation, that is, for every $a \in L, a \wedge a^{\prime}=0$ and $a \vee a^{\prime}=1$. For such algebras an endomorphism is a lattice endomorphism $\varphi$ that preserves', that is, $(a \varphi)^{\prime}=a^{\prime} \varphi$ for all $a \in L$. Every lattice with complementation is bounded, and any such endomorphism preserves 0 and 1.

The second result is that every monoid is isomorphic to the endomorphism semigroup of a lattice with complementation.

Both these results are consequences of much stronger theorems proved in this paper.

The proofs use some nontrivial graph theoretic and lattice theoretic results. For the reader's convenience, these are described in detail in $\S \S 2$ and 3 . Bounded lattices are dealt with in $\S 4$, and lattices with complementation in $\$ 5$. Some further results and

1 An alternative approach is investigated in [11]. 
open problems complete the discussion in $\S 6$.

2. Graphs, A graph $\langle X ; R\rangle$ consists of a set $X$ and a set $R$ of two-element subsets of $X$. (This is the definition of an unoriented graph; we do not need oriented graphs.) The elements of $X$ are called vertices, and the elements of $R$ are called edges. A compatible mapping $\varphi$ from the graph $\langle X ; R\rangle$ into the graph $\langle Y ; S\rangle$ is a map of $X$ into $Y$ satisfying: $\{a, b\} \in R$ implies $\{a \varphi, b \varphi\} \in S$.

We shall denote by $\boldsymbol{G}$ the following category of graphs:

The objects of $G$ are graphs $\langle X ; R\rangle$ satisfying conditions (1) and (2):

(1) for every $x \in X$ there is a seven element subset $\left\{x_{0}, x_{1}, \cdots, x_{6}\right\}$ of $X$ such that $x=x_{0}$, and $\left\{x_{0}, x_{1}\right\},\left\{x_{1}, x_{2}\right\}, \cdots,\left\{x_{5}, x_{6}\right\},\left\{x_{6}, x_{0}\right\} \in R$; in other words, every vertex lies on a cycle of length seven.

(2) there is no cycle of length less than seven.

The morphisms of $G$ are all the compatible maps.

Our constructions are based on the following result, which follows easily from the papers [7] and [8] of Z. Hedrlín and A. Pultr:

THEOREM 1. Every category of algebras is isomorphic to a full subcategory of $G$.

In more detail, this states the following. Let $K$ be a class of algebras of a fixed type (see, e.g., [2]), and let $\boldsymbol{K}$ also denote the category whose objects are the algebras in $\boldsymbol{K}$ and the morphisms are all homomorphisms. A functor $F: K \rightarrow G$ assigns to every algebra $\mathfrak{U} \in \boldsymbol{K}$ a graph $F(\mathfrak{U})$ and to every homomorphism $\varphi$ of $\mathfrak{U}$ into $\mathfrak{B}(\mathfrak{Z}, \mathfrak{B} \in \boldsymbol{K})$ a compatible map $F(\varphi)$ of $F(\mathfrak{C})$ into $F(\mathfrak{B})$ 'such that if $\varphi \psi=\alpha$, then $F(\varphi) F(\psi)=F(\alpha)$. A functor $F$ is called a full embedding if $F$ is one-to-one on objects, one-to-one on morphisms, and, in addition, it is full, that is, every morphism $\psi: F(\mathfrak{U}) \rightarrow$ $F(\mathfrak{B})$ in $G$ is of the form $F(\varphi)$ for some homomorphism $\varphi$ of $\mathfrak{A}$ into $\mathfrak{B}$.

Using these concepts Theorem 1 can be restated as follows: There exists a full embedding of $\boldsymbol{K}$ into $\boldsymbol{G}$ for every category $\boldsymbol{K}$ of algebras.

Since every monoid $M$ can be represented as the endomorphism semigroup of some algebra (see, e.g., [2]; the algebra can be constructed on the set $M$ by defining as operations left multiplications by elements of $M$ ), Theorem 1 contains as a special case the statement that every monoid can be represented as the semigroup of all compatible maps of a graph $\langle X ; R\rangle$ into itself, where $\langle X$; $R\rangle$ satifies (1) and (2).

3. Lattices. We denote by $\boldsymbol{L}$ the category of all bounded 
lattices with 0 and 1 preserving homomorphisms as morphisms.

Let $\mathfrak{n}$ be a cardinal. We identify cardinals with initial ordinals. A lattice with n-complementations is an algebra

$$
\left\langle L ; \wedge, \vee, 0,1, c_{0}, c_{1}, \cdots, c_{\gamma}, \cdots\right\rangle, \gamma<\mathfrak{n},
$$

where $\langle L ; \wedge, \vee, 0,1\rangle$ is a bounded lattice and for each $\gamma<\mathfrak{n}, a \in L$, $c_{\gamma}(a)$ is a complement of $a$. We shall denote by $\boldsymbol{L}_{\mathfrak{n}}$ the category whose objects are lattices with $\mathfrak{n}$ complementations satisfying conditions (3)-(5):

(3) $c_{i}\left(c_{i}(a)\right)=a$ for all $a \in L ; i<\mathfrak{n}\left(c_{i}\right.$ is an involution);

(4) $c_{i}\left(c_{j}(a)\right)=c_{j}\left(c_{i}(a)\right)$ for all $a \in L, i, j<\mathfrak{n}$ (the $c_{i}$ commute);

(5) for $a \in L, a \neq 0,1$, and $i, j<\mathfrak{n}, i \neq j, c_{i}(a) \neq c_{j}(a)$. The morphisms of $\boldsymbol{L}_{\mathfrak{n}}$ are lattice homomorphisms $\varphi$ preserving all $c_{i}, i<\mathfrak{n}$ (that is, $\left.c_{i}(a \varphi)=c_{i}(a) \varphi\right)$.

For a bounded lattice $L$ set

$$
C(L)=\{\{a, b\} \mid\{a, b\} \subseteq L, a \wedge b=0, a \vee b=1\},
$$

that is, $C(L)$ is the set of complemented pairs of $L$. The lattice $L$ is said to have incomparable complements if

$$
\{a, b\},\{a, c\} \in C(L) \text { and } b \geqq c \text { imply that } b=c .
$$

The lattice theoretic result we need is the following theorem of C. C. Chen and G. Grätzer [1]:

THeOREM 2. Let $M$ be a bounded lattice with incomparable complements, and let $A$ be a set disjoint with $M$. Let $\langle A \cup(M-\{0,1\}) ; C\rangle$ be a graph satisfying the following condition:

(6) $\{a, b\},\{a, c\} \in C, b, c \in M$, and $b \geqq c$ imply that $b=c$, and $\{a, b\} \in C, a \in M$ implies that $b \in A$.

Let $L$ be the bounded lattice freely generated by $M \cup A$, and denote by $\Theta[C]$ the smallest congruence relation on $L$ satisfying

(7) $\{a, b\} \in C$ implies that $a \wedge b \equiv 0(\Theta[C])$ and $a \vee b \equiv 1(\Theta[C])$. Let $M(A, C)=L / \Theta[C]$, and identify $a \in M \cup A$ with the congruence class containing it. Then

(8) $M$ is a $\{0,1\}$-sublattice of $M(A, C)$;

(9) $A \subseteq M(A, C)$;

(10) $A \cap M=\varnothing$ in $M(A, C)$;

(11) $M(A, C)$ is generated by $A \cup M$;

(12) $M(A, C)$ has incomparable complements;

(13) $C(M(A, C))=C(M) \cup C$.

4. Full embedding of $G$ into $L$. In this section we construct 
the functor $M$ which is the required full embedding. First we define $M$ on the objects.

Let $\langle X ; R\rangle$ be a graph in $G$. We apply Theorem 2 in the following way: let $M=\{0,1\}, A=X$, and $C=R$. Then $L$ is the free bounded lattice $L(X)$ generated by $X . M(X, R)$ has the following properties by Theorem 2:

(14) $X \cong M(X, R)$;

(15) $X$ generates $M(X, R)$;

(16) $C(M(X, R))=R \cup\{\{0,1\}\}$.

We define $M$ on $\langle X ; R\rangle$ to be $M(X, R)$. Let $\pi(X, R)$ denote the natural homomorphism of $L(X)$ onto $^{-} M(X, R)$ (with $\Theta[R]$ as induced congruence relation).

To define $M$ on morphisms, let $\varphi: X \rightarrow Y$ be a compatible map from $\langle X, R\rangle$ into $\langle Y$; $S\rangle$ in $G$. Then $\varphi$ has a natural extension to a homomorphism $L(\varphi)$ of $L(X)$ into $L(Y)$ (see the diagram). Let $\Theta$

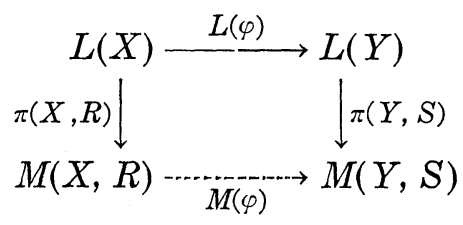

be the congruence relation induced by $L(\varphi) \pi(Y, S)$ (that is, for $a, b \in L(X), a \equiv b(\Theta)$ is equivalent to $a L(\varphi) \pi(Y, S)=b L(\varphi) \pi(Y, S))$. If $\{a, b\} \in R$, then (since $\varphi$ is compatible) $\{a \varphi, b \varphi\} \in S$, hence $a L(\varphi) \pi(Y, S)$ is a complement of $b L(\varphi) \pi(Y, S)$. Thus $\Theta$ is a congruence relation under which $a \wedge b \equiv 0(\Theta)$ and $a \vee b \equiv 1(\Theta)$ for any $\{a, b\} \in R$. By the definition of $\Theta[R]$ we conclude that $\Theta[R] \subseteq \Theta$. Hence by the Second Isomorphism Theorem (see e.g. [2, Th. 11.4]) there is a unique homomorphism $M(\varphi)$ such that $L(\varphi) \pi(Y, S)=\pi(X, R) M(\varphi)$. We define $M(\varphi)$ to be the value of $M$ at $\varphi$.

It is easy to check that $M$ is a functor from $G$ into $L$, and $M$ is one-to-one on objects. Since $X \subseteq M(X, R)$, and $M(\varphi)$ restricted to $X$ is $\varphi$, we get that $M$ is one-to-one on morphisms.

To prove that $M$ is full, let $\psi$ be a homomorphism of $M(X, R)$ into $M(Y, S)$. Take $x \in X$; we wish to show that $x \psi \in Y$. Recall that by (1) and (16) there exist $x_{0}=x, x_{1}, \cdots, x_{6} \in X$ such that $\left\{x_{0}, x_{1}\right\},\left\{x_{1}, x_{2}\right\}, \cdots,\left\{x_{5}, x_{6}\right\} \in C(M(X, R))$. Therefore,

$$
\left\{x_{0} \psi, x_{1} \psi\right\}, \cdots,\left\{x_{6} \psi, x_{0} \psi\right\} \in C(M(Y, S)) .
$$

By (16) this is possible only if $x_{0} \psi, \cdots, x_{8} \psi \in Y \cup\{0,1\}$; so $x_{0} \psi \in Y \cup\{0,1\}$. We claim that $x_{0} \psi \in Y$. Indeed, otherwise $x_{0} \psi \in\{0,1\}$, say $x_{0} \psi=0$. Then $x_{1} \psi=1$ (as the only complement of 0 is 1 ), $x_{2} \psi=0, x_{3} \psi=1$, $x_{4} \psi=0, x_{5} \psi=1, x_{6} \psi=0, x_{0} \psi=1$, a contradiction. Thus $\psi$ restricted 
to $X$ maps $X$ into $Y$; let $\varphi$ denote this map. Then $\varphi$ is a compatible map between $\langle X ; R\rangle$ and $\langle Y ; S\rangle$ and $M(\varphi)=\psi$ follows from (15).

Combining this with Theorem 1 we have

THEOREM 3. Every category of algebras can be fully embedded into the category $\boldsymbol{L}$ of bounded lattices. In particular, every monoid is isomorphic to the endomorphism semigroup of some bounded lattice.

5. Full embedding of $G$ into $L_{\mathrm{n}}$. We want to construct the functor $N_{\mathfrak{n}}$ which fully embeds $G$ into $L_{\mathfrak{n}}$. To outline the idea of the proof let us take the case $\mathfrak{n}=1$ (this eliminates the necessity to consider condition (4)). Let $\langle X ; R\rangle$ be a graph in $\boldsymbol{G}$, then $M_{0}=$ $M(X, R)$ has the same endomorphism semigroup as $\langle X ; R\rangle$. We define for every $a \in M_{0}, a \neq 0,1$ a new element $c(a)$, and apply Theorem 2 with $M=M_{0}, A=\left\{c(a) \mid a \in M_{0}, a \neq 0,1\right\}$, and

$$
C=\left\{\{a, c(a)\} \mid a \in M_{0}, a \neq 0,1\right\} .
$$

Then we define $c$ on $M_{1}=M(A, C)$ as follows: $c(0)=1, c(1)=0$, for $a \in M_{0}, a \neq 0,1, c(a)$ is the new element, $c(c(a))=a$, and $c(a)$ is not defined for any other element. Then we define $A_{1}=\left\{c(a) \mid a \in M_{1}\right.$, $c(a)$ is not defined $\}$ to get $M_{2}$, and so on. $N_{1}(X, R)$ will be $\mathrm{U}\left(M_{i} \mid i<\omega\right)$. The endomorphisms will work out all right because the elements of $X$ are still distinguished by being on seven cycles of complementary elements.

Now we proceed with a formal construction of the functor $N_{\mathfrak{n}}$.

Recall that $\mathfrak{n}$ is identified with the initial ordinal of cardinality $\mathfrak{n}$, hence $\mathfrak{n}$ is a set of ordinals. Let $E_{\mathfrak{n}}$ denote the set of all finite subsets of $\mathfrak{t}$. For $a, b \in E_{\mathrm{n}}$ let $a+b$ denote the symmetric difference. We define a graph $\left\langle E_{\mathfrak{n}} ; D_{\mathfrak{n}}\right\rangle$, where $\{a, b\} \in D_{\mathfrak{n}}$ if and only if $|a+b|=1$. Finally, for $a \in \mathfrak{n}$ we define the function $\varepsilon_{a}: E_{\mathfrak{n}} \rightarrow E_{\mathfrak{n}}$ by

$$
\varepsilon_{a}(x)=\left\{\begin{array}{l}
x \cup\{a\} \text { if } a \notin x \\
x-\{a\} \text { if } a \in x
\end{array}\right.
$$

Let us make a few observations about the $\varepsilon_{a}$ :

(17) $\varepsilon_{a}\left(\varepsilon_{a}(x)\right)=x$ for all $x \in E_{\mathfrak{n}}$;

(18) $\varepsilon_{a}\left(\varepsilon_{b}(x)\right)=\varepsilon_{b}\left(\varepsilon_{a}(x)\right)$ for all $x \in E_{\mathfrak{n}}, a \neq b$;

(19) $\varepsilon_{a}(x) \neq \varepsilon_{b}(x)$ for all $x \in E_{n}, a \neq b$;

(20) if $x=\left\{a_{1}, \cdots, a_{\mathfrak{n}}\right\}$, then $\varepsilon_{a_{1}}\left(\varepsilon_{a_{2}}\left(\cdots\left(\varepsilon_{a_{\mathfrak{n}}}(\phi) \cdots\right)\right)=x\right.$;

(21) all cycles in $\left\langle E_{n} ; D_{n}\right\rangle$ are of even length.

All these are obvious; to prove (21) observe that $\left|\varepsilon_{a}(x)\right|=|x| \pm 1$.

For $\langle X ; R\rangle$ in $G$ set $M_{0}=M(X, R)$ as defined in $\S 4$. We define a graph $\left\langle\left(M_{0}-\{0,1\}\right) \times E_{n} ; C_{1}\right\rangle$ by 


$$
C_{1}=\left\{\left\langle\left\langle a, e_{1}\right\rangle,\left\langle a, e_{2}\right\rangle\right\} \mid a \in M_{0}-\{0,1\}, e_{1}, e_{2} \in E_{\mathfrak{n}},\left\{e_{1}, e_{2}\right\} \in D_{\mathfrak{n}}\right\} .
$$

Identifying $a \in M_{0}-\{0,1\}$ with $\langle a, \varnothing\rangle$ we can apply Theorem 2 to $M_{0},\left(M_{0}-\{0,1\}\right) \times\left(E_{\mathrm{n}}-\{\varnothing\}\right)$, and $C_{1}$; to see that we only have to check (6) for $C_{1}$ : if $b, c \in M,\{a, b\},\{a, c\} \in C_{1}$, and $b \geqq c$, then $b=$ $\langle b, \varnothing\rangle, \quad c=\langle c, \varnothing\rangle, \quad a=\left\langle b, e_{1}\right\rangle=\left\langle c, e_{1}\right\rangle$, hence $b=c$. Thus by Theorem 2 we get the lattice $M_{1}=L(X) / \Theta\left[C_{1}\right]$.

For all $a \in \mathfrak{n}$ we define $c_{a}$ on a part of $M_{1}$ :

(22) $c_{a}(\langle b, e\rangle)=\left\langle b, \varepsilon_{a}(e)\right\rangle$,

(23) $c_{a}(0)=1, c_{a}(1)=0$.

Observe that $c_{a}\left(c_{a}(x)\right)=x$ by (17), $c_{a}\left(c_{b}(x)\right)=c_{b}\left(c_{a}(x)\right)$ by (18). Also, $c_{a}(x)$ is a complement of $x$ because $\left|e+\varepsilon_{a}(e)\right|=1$, hence

$$
\left\{\langle b, e\rangle,\left\langle b, \varepsilon_{a}(e)\right\rangle\right\} \in C_{1} ;
$$

finally, by (13), $C\left(M_{1}\right)=C(M) \cup C_{1}$. Note that $c_{a}$ is defined exactly on $M_{0} \cup C_{1}$. Let $\bar{M}_{1}$ be $M_{1}$ with all the $c_{a}, a \in \mathfrak{H}$ as partial unary operations.

Now assume that $M_{0}, M_{1}, \cdots, M_{i}, \bar{M}_{i}$ have already been defined and $M_{i}$ has no comparable complements. Let $A_{i}$ be the subset of $M_{i}$ consisting of all noncomplemented elements of $M_{i}$. Then we form the set $\left(M_{i}-A_{i}\right) \cup\left(A_{i} \times E_{\mathrm{n}}\right)$; by identifying $a \in A$ with $\langle a, \varnothing\rangle, M_{i}$ is a subset of this set. Define $C_{i+1}=\left\{\left\langle\left\langle a, e_{1}\right\rangle,\left\langle a, e_{2}\right\rangle\right\} \mid a \in A_{i},\left\{e_{1}, e_{2}\right\} \in D_{\mathfrak{1 1}}\right\}$. Again we apply Theorem 2 to $M_{i},\left(A_{i}-\{\varnothing\}\right) \times E_{n}$, and $C_{i+1}$, getting the lattice $M_{i+1}$. We define $c_{a}(a \in \mathfrak{n})$ as a partial unary operation on $M_{i+1}$ as follows: for the complemented elements of $M_{i}$ we have already defined $c_{a}$; if $x \in M_{i}$, but $x$ is not complemented, then $x \in A_{i}$ and we set $c_{a}(x)=\langle x,\{a\}\rangle$; in general $c_{a}(\langle x, e\rangle)=\left\langle x, \varepsilon_{a}(e)\right\rangle$. This defines $c_{a}$ for all complemented elements of $M_{i+1}$. Let $\bar{M}_{i+1}$ denote the $M_{i+1}$ with all the $c_{a}$.

Finally, we set

$$
N_{\mathrm{n}}(\langle X ; R\rangle)=\mathbf{U}\left(M_{i} \mid 1 \leqq i<\omega\right) .
$$

Observe that $\bar{M}_{\omega}=\bigcup\left(\bar{M}_{i} \mid i<\omega\right)$ is an algebra defined on the bounded lattice $M_{\omega}=\bigcup\left(M_{i} \mid i<\omega\right)$. Since the domain of $c_{a}$ in $M_{i+1}$ included $M_{i}, c_{a}$ is fully defined on $M_{\omega}$. Thus $\bar{M}_{\omega}$ is a bounded lattice with $\mathfrak{t}$ complementations. It is clear from (17)-(19) that in fact (3)-(5) are satisfied, that is, $\bar{M}_{\omega}$ is in $\boldsymbol{L}_{\mathfrak{n}}$.

The next task is to construct $N_{\mathfrak{n}}$ on morphisms. To facilitate this we need a special case of a result of [4], which we shall give as a corollary to Theorem 2:

Corollary to Theorem 2. Let $M, A, C$, and also $M_{1}, A_{1}, C_{1}$ satisfy the condition of Theorem 2. Let $\phi$ be a map of $M \cup A$ into 
$M_{1} \cup A_{1}$ such that $M \varphi \subseteq M_{1}, \varphi$ is a homomorphism of $M$ into $M_{1}$, and $\{a, b\} \in C$ implies that $\{a \varphi, b \varphi\} \in C_{1}$. Then there exists a unique extension of $\varphi$ to a homomorphism $\bar{\phi}$ of $M(A, C)$ into $M\left(A_{1}, C_{1}\right)$.

Now let $\varphi: X \rightarrow Y$ be a compatible map of $\langle X ; R\rangle$ into $\langle Y$; $S\rangle$, and let $\bar{M}_{0}, \bar{M}_{1}, \cdots$ be the sequence of lattices with complementations constructed from $\langle X ; R\rangle$ and $\bar{P}_{0}, \bar{P}_{1}, \ldots$ the sequence for $\langle Y ; S\rangle$. We shall construct mappings $\varphi_{i}: M_{i} \rightarrow P_{i}$. In $\S 4$ we constructed a homomorphism $\varphi_{0}: M_{0} \rightarrow P_{0}$ extending $\varphi$. We extend $\varphi_{0}$ to

$$
M_{0} \cup\left(\left(M_{0}-\{0,1\}\right) \times\left(E_{\mathfrak{n}}-\{\varnothing\}\right)\right)
$$

as follows:

$$
\langle b, e\rangle \bar{\varphi}_{0}= \begin{cases}\left\langle b \varphi_{0}, e\right\rangle & \text { if } b \varphi_{0} \neq 0,1 ; \\ 0 & \text { if } b \varphi_{0}=0 \text { and }|e| \text { is even, or } b \varphi_{0}=1 \text { and }|e| \text { is odd; } \\ 1 & \text { if } b \varphi_{0}=1 \text { and }|e| \text { is even, or } b \varphi_{0}=0 \text { and }|e| \text { is odd . }\end{cases}
$$

Clearly, $\bar{\phi}_{0}$ maps $M_{0} \cup\left(\left(M_{0}-\{0,1\}\right) \times\left(E_{\mathrm{n}}-\{\varnothing\}\right)\right)$ into

$$
P_{0} \cup\left(\left(P_{0}-\{0,1\}\right) \times\left(E_{\mathrm{n}}-\{\varnothing\}\right)\right)
$$

and it satisfies the requirements of the Corollary to Theorem 2, hence it can be extended to a homomorphism $\varphi_{1}$ of $M_{1}$ into $P_{1}$. It is also clear, that for all $x \in M_{1}, c_{a}(x) \varphi_{1}=c_{a}\left(x \varphi_{1}\right)$, provided $c_{a}(x)$ is defined. Hence $\varphi_{1}$ is a homomorphism of $\bar{M}_{1}$ into $\bar{P}_{1}$. Thus applying the Corollary to Theorem 2 we get all the $\varphi_{i}$, and $\bar{\varphi}=\mathbf{U}\left(\varphi_{i} \mid i<\omega\right)$ is defined to be $N_{\mathfrak{n}}(\varphi)$. This completes the definition of $N_{\mathfrak{n}}$.

To show that $N_{\mathfrak{n}}$ is a functor one can use a direct computation or employ an argument similar to the one we used for $M$. Namely, one can represent $N_{\mathfrak{n}}(\langle X ; R\rangle)$ as a quotient structure $F(X, R) / \theta[R]$, where $F(X, R)$ is the free m-complemented lattice generated by $M(X, R)$ and $\Theta[R]$ is the smallest congruence relation forced by (3) and (4). (Note that (5) will be satisfied by $F(X, R) / \Theta[R]$.) Then we get $N_{11}(\varphi)$ by a diagram similar to the one in $\S 4$ which implies that $N_{\mathrm{n}}$ is a functor.

$N_{\mathfrak{n}}$ is one-to-one on objects and maps since $X$ generates $N_{\mathfrak{n}}(\langle X ; R\rangle)$. It remains to show that $N_{\mathrm{n}}$ is full. Let $\varphi$ be a homomorphism of $N_{\mathfrak{n}}(\langle X ; R\rangle)$ into $N_{\mathfrak{n}}(\langle Y ; S\rangle)$ just as in $\S 4$ it is enough to show that $X \varphi \subseteq Y$. Every $x \in X$ lies on a cycle of complementary pairs of length seven. Hence $x \varphi$ lies on a quotient of a cycle of length seven, which is either a cycle of length seven, and in this case $x \varphi \in Y$ by (21), or the quotient contains a cycle of length 3 or 5 , contradicting (2) and (21).

Combining the full embedding $N_{\mathfrak{n}}$ with the one given in Theorem 1 we conclude 
THEOREM 4. Let $\mathfrak{n}$ be a cardinal. Any category of algebras is isomorphic to a full subcategory of $\boldsymbol{L}_{\mathfrak{n}}$. Consequently, every monoid is isomorphic to the endormorphism semigroup of a lattice with $\mathfrak{n}$ complementations.

6. Concluding remarks. Utilizing the fact that $|M(X, R)|=$ $|X|$ provided that $|X|$ is infinite, and that $\left|N_{\mathfrak{n}}(X, R)\right|=|X|$ provided that $|X| \geqq \mathfrak{n}+\boldsymbol{\aleph}_{0}$ we can combine Theorems 3 and 4 with the results of [9] to get the number of nonisomorphic objects in $L$ and $L_{\mathfrak{n}}$ with a given endomorphism semigroup.

THEOREM 5. Let $S$ be a monoid with $\mathfrak{p}$ elements and let $\mathfrak{m}$ be an infinite cardinal with $\mathfrak{m} \geqq \mathfrak{p}$. Then there are exactly $2^{\mathfrak{m}}$ pairwise nonisomorphic bounded lattices (and bounded lattices with $\mathfrak{n}$ complementation satisfying (3)-(5), provided that $\mathfrak{n} \leqq \mathfrak{m})$ of cardinality $\mathfrak{m}$ whose endomorphism semigroup is isomorphic to $S$.

Note that Theorem 5 includes the statement that $S$ can be represented as an endormorphism semigroup of arbitrarily large bounded lattices.

The method of this paper is not applicable, however, to get results on the endomorphism semigroups of lattices of finite length. Indeed, $M(X, R)$ and $N_{\mathrm{n}}(X, R)$ are of infinite length unless $\langle X ; R\rangle$ is very close to the complete graph. A new method to deal with this problem is proposed in [10] which yields, for instance, that every group is isomorphic to the endormorphism semigroup of a lattice of finite length (which also happens to be complemented).

Note that the functors $M$ and $N_{\mathfrak{n}}$ take onto maps to onto maps, but almost none of the one-to-one maps are carried into one-to-one maps.

Problem 1. Characterize the endomorphism semigroups of lattices of finite length.

Problem 2. For what categories $\boldsymbol{K}$ of algebras does there exist a full embedding of $\boldsymbol{K}$ into $\boldsymbol{L}$ and $\boldsymbol{L}_{\mathrm{n}}$ which takes one-to-one maps into one-to-one maps. Could such full embeddings be constructed that take onto maps to onto maps?

Problem 3. Characterize the endomorphism semigroups of complemented lattices.

Problem 4. Find classes of monoids for which the conclusion of Theorem 5 holds under the assumption $\mathfrak{p}<\mathfrak{n} \leqq 2^{\mathfrak{p}}$ ? 


\section{REFERENCES}

1. C. C. Chen and G. Grätzer, On the construction of complemented lattices, J. Algebra 11 (1969), 56-63.

2. G. Grätzer, Universal Algebra, The University Series in Higher Mathematics.

D. Van Nostrand Co. Inc., Princeton, N. J., 1968.

3. L Lectures on Lattice Theory, Vol. 1., W. H. Freeman and Company, San Francisco, Calif. (to appear in 1971).

4. - A reduced free product of lattices, Fund. Math. (to appear).

5. Z. Hedrlín and J. Lambek, How comprehensive is the category of semigroups? J. Alg. 11 (1969), 195-212.

6. Z. Hedrlín and R. H. McDowell, Partly ordered sets with given monoid of orderpreserving map (to appear).

7. Z. Hedrlín and A. Pultr, Relations (graphs) with given infinite semigroups, Monatsh. Math. 68 (1964), 421-425.

8. Z. Hedrlín and A. Pultr, On full embeddings of categories of algebras, Illinois J. Math. 10 (1966), 392-406.

9. Z. Hedrlín and J. Sichler, Any boundable binding category contains a proper class of mutually disjoint copies of itself (to appear).

10. R. McKenzie and J. Sichler, Endomorphisms of finite complemented lattices and of lattices of finite length (to appear).

11. J. Sichler, Every monoid is isomorphic to the monoid of all non-constant endomorphisms of a lattice (to appear).

Received April 21, 1970. The work of both authors was supported by the National Research Council of Canada.

The University of Manitoba

Winnipeg, Manitoba, Canada 



\section{PACIFIC JOURNAL OF MATHEMATICS}

\section{EDITORS}

\author{
H. SAMELSON \\ Stanford University \\ Stanford, California 94305 \\ Richard Pierce \\ University of Washington \\ Seattle, Washington 98105
}

\author{
J. DugundJI \\ Department of Mathematics \\ University of Southern California \\ Los Angeles, California 90007 \\ RICHARD ARENS \\ University of California \\ Los Angeles, California 9.0024
}

\section{ASSOCIATE EDITORS}

\begin{tabular}{|c|c|}
\hline E. F. BECKENBACH & K. YoshidA \\
\hline \multicolumn{2}{|c|}{ SUPPORTING INSTITUTIONS } \\
\hline UNIVERSITY OF BRITISH COLUMBIA & STANFORD UNIVERSITY \\
\hline CALIFORNIA INSTITUTE OF TECHNOLOGY & UNIVERSITY OF TOKYO \\
\hline UNIVERSITY OF CALIFORNIA & UNIVERSITY OF UTAH \\
\hline MONTANA STATE UNIVERSITY & WASHINGTON STATE UNIVERSITY \\
\hline UNIVERSITY OF NEVADA & UNIVERSITY OF WASHINGTON \\
\hline NEW MEXICO STATE UNIVERSITY & $* \quad * \quad *$ \\
\hline OREGON STATE UNIVERSITY & AMERICAN MATHEMATICAL SOCIETY \\
\hline UNIVERSITY OF OREGON & CHEVRON RESEARCH CORPORATION \\
\hline OSAKA UNIVERSITY & TRW SYSTEMS \\
\hline UNIVERSITY OF SOUTHERN CALIFORNIA & NAVAL WEAPONS CENTER \\
\hline
\end{tabular}

The Supporting Institutions listed above contribute to the cost of publication of this Journal, but they are not owners or publishers and have no responsibility for its content or policies.

Mathematical papers intended for publication in the Pacific Journal of Mathematics should be in typed form or offset-reproduced, (not dittoed), double spaced with large margins. Underline Greek letters in red, German in green, and script in blue. The first paragraph or two must be capable of being used separately as a synopsis of the entire paper. The editorial "we" must not be used in the synopsis, and items of the bibliography should not be cited there unless absolutely necessary, in which case they must be identified by author and Journal, rather than by item number. Manuscripts, in duplicate if possible, may be sent to any one of the four editors. Please classify according to the scheme of Math. Rev. Index to Vol. 39. All other communications to the editors should be addressed to the managing editor, Richard Arens, University of California, Los Angeles, California, 90024.

50 reprints are provided free for each article; additional copies may be obtained at cost in multiples of 50 .

The Pacific Journal of Mathematics is published monthly. Effective with Volume 16 the price per volume (3 numbers) is $\$ 8.00$; single issues, $\$ 3.00$. Special price for current issues to individual faculty members of supporting institutions and to individual members of the American Mathematical Society: $\$ 4.00$ per volume; single issues $\$ 1.50$. Back numbers are available.

Subscriptions, orders for back numbers, and changes of address should be sent to Pacific Journal of Mathematics, 103 Highland Boulevard, Berkeley, California, 94708.

PUBLISHED BY PACIFIC JOURNAL OF MATHEMATICS, A NON-PROFIT CORPORATION

Printed at Kokusai Bunken Insatsusha (Internatıonal Academic Printing Co., Ltd.), 7-17, Fujimi 2-chome, Chiyoda-ku, Tokyo, Japan. 


\section{Pacific Journal of Mathematics}

\section{Vol. 35, No. $3 \quad$ November, 1970}

John D. Arrison and Michael Rich, On nearly commutative degree one algebras . . . 533

Bruce Alan Barnes, Algebras with minimal left ideals which are Hilbert spaces . . . . 537

Robert F. Brown, An elementary proof of the uniqueness of the fixed point index . . . 549

Ronn L. Carpenter, Principal ideals in F-algebras .................... 559

Chen Chung Chang and Yiannis (John) Nicolas Moschovakis, The Suslin-Kleene

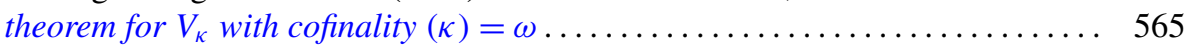

Theodore Seio Chihara, The derived set of the spectrum of a distribution

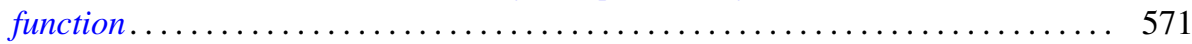

Tae Geun Cho, On the Choquet boundary for a nonclosed subspace of $C(S) \ldots \ldots \quad 575$

Richard Brian Darst, The Lebesgue decomposition, Radon-Nikodym derivative,

conditional expectation, and martingale convergence for lattices of sets .......

David E. Fields, Dimension theory in power series rings . . . . . . . . . . . .

Michael Lawrence Fredman, Congruence formulas obtained by counting

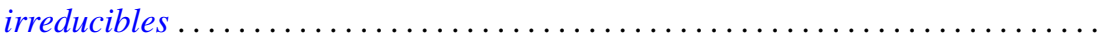

John Eric Gilbert, On the ideal structure of some algebras of analytic functions.....

G. Goss and Giovanni Viglino, Some topological properties weaker than

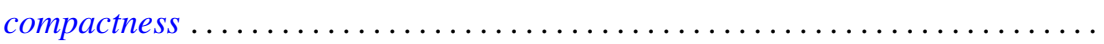

581

601

625

George Grätzer and J. Sichler, On the endomorphism semigroup (and category) of

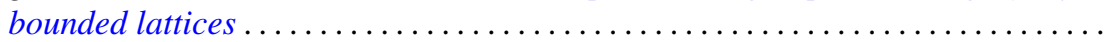

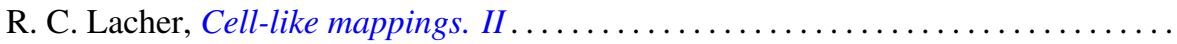

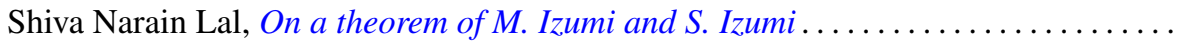

661

Howard Barrow Lambert, Differential mappings on a vector space ...............

Richard G. Levin and Takayuki Tamura, Notes on commutative power joined

semigroups.

Robert Edward Lewand and Kevin Mor McCrimmon, Macdonald's theorem for quadratic Jordan algebras.

J. A. Marti, On some types of completeness in topological vector spaces ....

Walter J. Meyer, Characterization of the Steiner point

717

Saad H. Mohamed, Rings whose homomorphic images are $q$-rings ...

727

Thomas V. O'Brien and William Lawrence Reddy, Each compact orientable surface

of positive genus admits an expansive homeomorphism ...

737

Robert James Plemmons and M. T. West, On the semigroup of binary relations...

743

Calvin R. Putnam, Unbounded inverses of hyponormal operator . .

755

William T. Reid, Some remarks on special disconjugacy criteria for differential

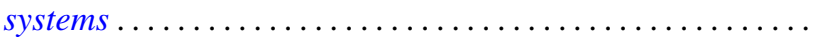

C. Ambrose Rogers, The convex generation of convex Borel sets in euclidean

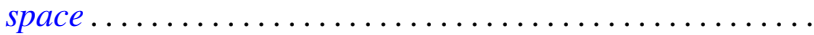

S. Saran, A general theorem for bilinear generating functions .

S. W. Smith, Cone relationships of biorthogonal systems ......

Wolmer Vasconcelos, On commutative endomorphism rings ....

795

Vernon Emil Zander, Products of finitely additive set functions from Orlicz

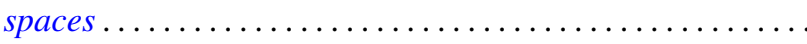

G. Sankaranarayanan and C. Suyambulingom, Correction to: "Some renewal

theorems concerning a sequence of correlated random variables" .

Joseph Zaks, Correction to: "Trivially extending decompositions of $E^{n}$ ”....... 805

Dong Hoon Lee, Correction to: "The adjoint group of Lie groups" ............ 805

James Edward Ward, Correction to: "Two-groups and Jordan algebras". 